\title{
Circuit
}

Musiques contemporaines

\section{Créé dans LeVivier}

\section{Cléo Palacio-Quintin}

Volume 22, numéro 2, 2012

URI : https://id.erudit.org/iderudit/1012796ar

DOI : https://doi.org/10.7202/1012796ar

Aller au sommaire du numéro

\section{Éditeur(s)}

Les Presses de l’Université de Montréal

\section{ISSN}

1183-1693 (imprimé)

1488-9692 (numérique)

Découvrir la revue

Citer ce compte rendu

Palacio-Quintin, C. (2012). Compte rendu de [Créé dans LeVivier]. Circuit, 22(2),

81-84. https://doi.org/10.7202/1012796ar

Ce document est protégé par la loi sur le droit d'auteur. L'utilisation des services d’Érudit (y compris la reproduction) est assujettie à sa politique d'utilisation que vous pouvez consulter en ligne.

https://apropos.erudit.org/fr/usagers/politique-dutilisation/
Cet article est diffusé et préservé par Érudit.

Érudit est un consortium interuniversitaire sans but lucratif composé de l’Université de Montréal, l’Université Laval et l’Université du Québec à Montréal. Il a pour mission la promotion et la valorisation de la recherche. https://www.erudit.org/fr/ 


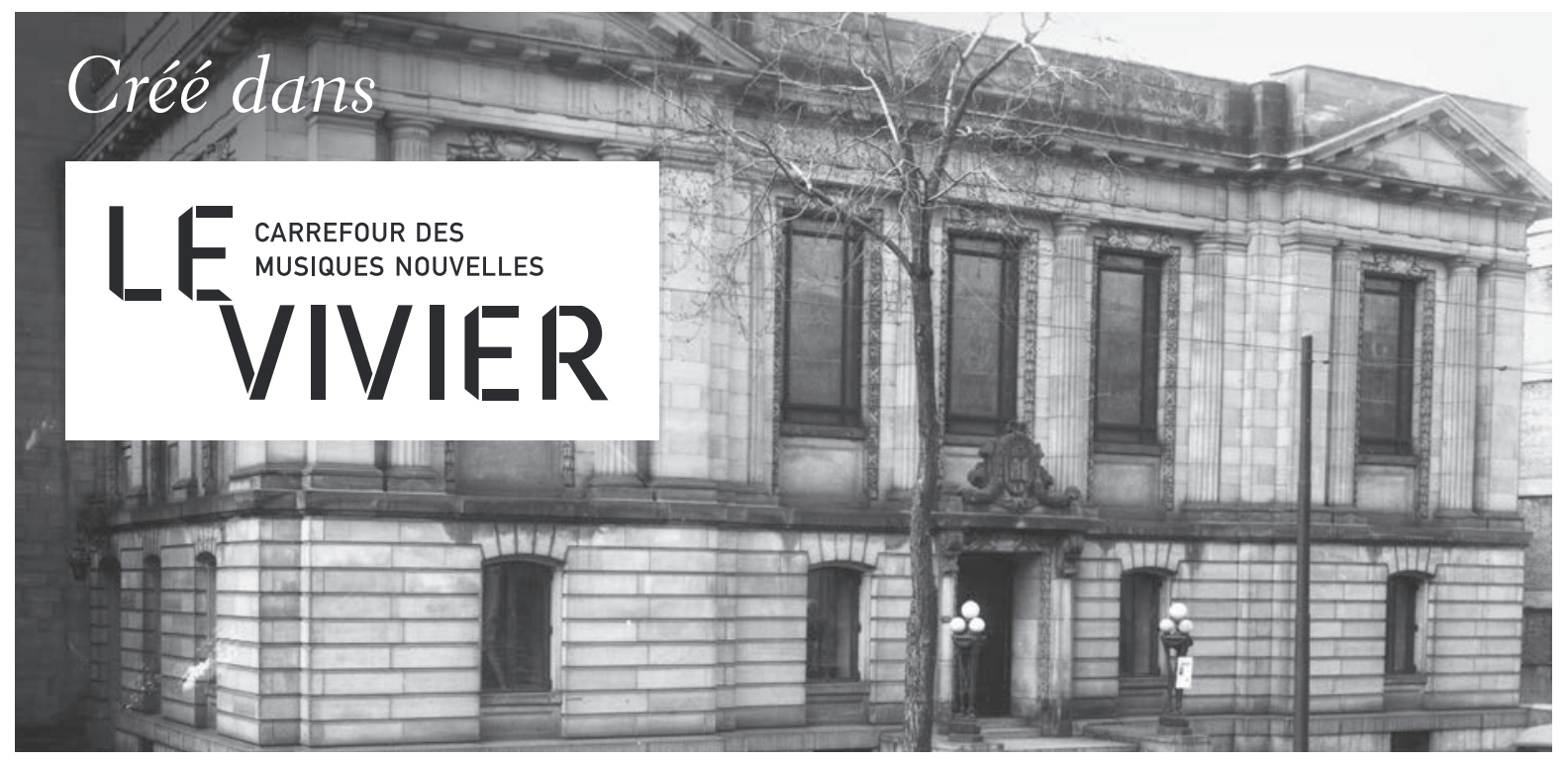

Cléo Palacio-Quintin

Photo : Archives de la Ville de Montréal.

Le Vivier est un diffuseur spécialisé, formé en 2007 de l'association de plus de 25 ensembles et organismes musicaux québécois. Sa principale mission est de favoriser le développement des musiques nouvelles et d'offrir à tous, par la diffusion d'œuvres de qualité, une porte ouverte sur la culture.

Les membres du Groupe Le Vivier désirent doter Montréal d'un lieu de création et de diffusion pour les musiques nouvelles. Le Vivier s'est vu confier l'occupation du magnifique immeuble patrimonial de l'ancienne Bibliothèque Saint-Sulpice, en plein cœur du Quartier des spectacles et du Quartier latin à Montréal. L'édifice, bientôt centenaire, doit cependant être rénové avant qu'il ne soit fonctionnel. La création de ce carrefour des musiques nouvelles va permettre une série d'actions culturelles destinées aux citoyens, aux familles, aux établissements scolaires et aux créateurs, et ainsi assurer la diffusion des musiques nouvelles dans toute leur diversité.

En attendant que les travaux de rénovation de l'ancienne Bibliothèque Saint-Sulpice soient achevés, Le Vivier poursuit son mandat de diffuseur en 
présentant une série de concerts itinérante à Montréal, en collaboration avec ses membres.

La revue Circuit veut mettre en valeur ces créateurs et interprètes, en présentant une liste des œuvres jouées en création dans le cadre de la série de concerts du Groupe Le Vivier. Pour débuter cette nouvelle chronique qui sera récurrente, voici donc par ordre chronologique, la liste des œuvres présentées en création lors de la saison artistique 2010-2011.

\section{- Saison artistique 2010-2011 -}

30 septembre 2010

Théâtre Paul-Desmarais Centre canadien d'architecture

28 octobre 2010 Studio Hydro-Québec Monument National

11 novembre 2010

Salle de concert - Conservatoire de musique de Montréal

24 novembre 2010

Salle Claude-Champagne Université de Montréal
Transmission $\bullet$ Xtc

JAMES HARLEY $\bullet$ TreDue

Guy Pelletier (flûte), Lori Freedman (clarinette), Alain Giguère (violon), Julie Trudeau (violoncelle), Julien Grégoire (percussions), Brigitte Poulin (piano)

Réseaux des arts médiatiques • Mathew Adkins / Martin Bédard MARTIN BÉDARD • Push \& Pull

Martin Bédard (diffusion électroacoustique)

Ensemble contemporain de Montréal ECM+

- Génération 10, Tournée canadienne

Simon Martin • Musique d'art pour orchestre de chambre

Christopher MaYo $\bullet$ Binding the Quiet

CASSANDRa MiLLer $\bullet$ Concerto for violin and blindfolded ensemble

(a set of extravagant competitions)

GORDON WilLIAMSON • Anticipated, emancipated

Véronique Lacroix (direction), Véronique Mathieu (violon solo)

Nouvel Ensemble Moderne • Forum 2010: Musique et vidéo d'art, Concert 1

TAYLOR BROOK • Les trains où vont les choses

RAFFAELE GRIMALDI • Agora Obscura

ESAIAS JäRNEGARD • Tagen-tiden-tingen

Lorraine Vaillancourt (direction) 
Nouvel Ensemble Moderne • Forum 2010: Musique et vidéo d'art, Concert 2

Juan de Dios Magdaleno $\bullet$ Yksi [kaksi]

Hugo Ribeiro $\bullet$ Nocturne: Rituel

Diego SoIFER • Trenes

Lu WANG • Cross-Around

PEIYING YUAN • Pemesu

Lorraine Vaillancourt (direction)

Codes d'accès $\bullet$ Son, espace, temps

Laurent Aglat • Mémoire des choses qui tombent

Hugues ClÉMent • Étude cyphotique

ThiERry GaUTHIER • Abstractions

Julien-Robert Legault-SaLVail $\bullet$ Video Loop

Julien Compagne (percussions), Laurent Aglat, Thierry Gauthier, Hugues Clément, Julien-Robert Legault-Salvail (diffusion)

Quatuor Molinari • Du neuf pour neuf

Michael Oesterle $\bullet$ Melodeon

ANDRÉ RISTIC • Variétés rupestres

Olga Ranzenhofer (violon), Frédéric Bednarz (violon),

Frédéric Lambert (alto), Pierre-Alain Bouvrette (violoncelle),

Danièle Bourget (flûte), Martin Carpentier (clarinette),

Normand Forget (hautbois), Mathieu Lussier (basson),

Louis-Philippe Marsolais (cor)

Productions SuperMusique • Géométries aléatoires

SANDEeP BhaGWati $\bullet$ Oiseaux d'ailleurs

Malcolm Goldstein • Espaces en mouvement

Diane Labrosse $\bullet$ Les cahiers

JESSE STEWART $\bullet$ Reinventing the Wheel

Magali Babin (électronique), Guido Del Fabbro (violon),

Jean Derome (saxophones, flûte, objets), Émilie Girard-Charest (violoncelle), Joane Hétu (saxophone alto, voix), Philippe Lauzier (clarinettes), Alexandre St-Onge (contrebasse, électronique), Pierre Tanguay (batterie), Scott Thomson (trombone), Danielle Palardy Roger (percussions)
26 novembre 2010

Salle Claude-Champagne -

Université de Montréal

$1^{\text {er }}$ décembre 2010

Ex-Centris

11 février 2011

Salle de concert - Conservatoire de musique de Montréal

22 mars 2011

Salle multimédia - Conservatoire de musique de Montréal 
19 mai 2011

Chapelle historique du Bon-Pasteur

Studio 303 - Édifice Belgo

5 juin 2011

Salle multimédia - Conservatoire de musique de Montréal
Quasar et Quatuor Arte (Suisse) • Alter Ego II

WOLF EdWARDS • Antanaklasi

Marie-Chantal Leclair (saxophone soprano), Mathieu Leclair (saxophone alto), André Leroux (saxophone ténor), Jean-Marc Bouchard (saxophone baryton), Beat Hofstetter (saxophone soprano), Sascha Armbruster (saxophone alto), Andrea Formenti (saxophone ténor), Beat Kappeler (saxophone baryton)

In Extensio • Urbanisme/Urbanité II

EMILY HALL • Long After the Night

Mariève Lauzon (flûte), Louise Campbell (clarinette),

Barah Héon-Morissette (percussions)

Productions Totem Contemporain • Totems électriques III

WOLF EDWARDS • Kerato

Gilles GOBEIL • Bol-Hydre

Charles Quevillon • Locustes

Jean-François Laporte (canette sifflante), Jean-Marc Bouchard (organophone), Marie-Chantal Leclair (orgue de sirènes), Gilles Gobeil (diffusion) 